\title{
Time Optimal Load Shedding for Distributed Power Systems
}

\author{
E. E. Aponte, Student Member, IEEE, and J. Keith Nelson, Fellow, IEEE
}

\begin{abstract}
The formulation and computation of an optimal load shedding time algorithm is presented. This new concept was developed as an enhancement to the application of optimal load shedding for corrective control to support islanded power systems. The methodology combines nonlinear mathematical programming and discretized differential-algebraic power systems equations to estimate the optimal amount of load to be shed as well as the best time to shed it. Several simulated scenarios are studied and results presented.
\end{abstract}

Index Terms-Distributed Generation, load shedding, optimization.

\begin{tabular}{|c|c|}
\hline & Notations \\
\hline$x$ & state variables vector \\
\hline$y$ & algebraic variables vector \\
\hline$u$ & control variables vector \\
\hline$n_{x}$ & number of state variables \\
\hline$n_{y}$ & number of algebraic variables \\
\hline$n_{T}$ & number of time steps \\
\hline$H_{k}$ & discretized transition equation \\
\hline$C$ & total cost of current iteration \\
\hline$C_{1}$ & cost associated with the state variables \\
\hline$C_{2}$ & cost associated with the algebraic variables \\
\hline$C_{3}$ & cost associated with the control input \\
\hline$Q$ & weights matrix \\
\hline$L$ & augmented cost function \\
\hline$\gamma$ & lagrangian multiplier, state constraints \\
\hline$\beta$ & lagrangian multiplier, network constraints \\
\hline$P_{L S_{i}}$ & load shedding fraction at bus $i$ \\
\hline$t_{1}$ & $\begin{array}{l}\text { load shedding time, measured after } \\
\text { formation of island }\end{array}$ \\
\hline
\end{tabular}

\section{INTRODUCTION}

$\mathbf{P}$ OWER systems exist to supply reliable electric energy to their customers. The structure of centralized systems relies heavily on transmission and distribution systems to transport energy from bulk production sites to load centers. The demand for electricity continues to grow and the current centralized model is becoming inadequate. Transmission congestion, generation shortages, environmental concerns and dependency on foreign resources are forcing the re-evaluation of the traditional strategy. Industry restructuring and long lead times for new major installations has also precipitated a change in thinking.

Distributed Generation (DG) may provide a new alternative. Under its full implementation, local resources like wind, solar,

Manuscript received Xxxxxxx xx, XXXX; revised XXXXXX xx, XXXX. J. K. Nelson is with the Rensselaer Polytechnic Institute.

E. E. Aponte is with the University of Puerto Rico at Mayaguez. hydrogen, and others could be harvested more effectively. Smaller facilities could be developed faster and with less regulatory intervention depending on the technology. Such initiatives could spark local interest into cleaner, environmentally friendly energy. Issues like location, interconnection, stability, protection, and security amongst others are being actively evaluated [1]-[6]. Some of the work on these areas is yielding promising results.

At the heart of the technical controversy lies the issue of islanding. When generation equipment is located within traditionally radial distribution systems, unintentional islands may result. These could have fatal consequences to linesmen and others who might not be aware of the danger. However, under certain conditions, the same techniques used to detect and eliminate unintentional islands might end up as potential sources of instability [7]. Under the current guidelines (IEEE P1547 [8]), generators connected to the power system are required to detect an islanded situation (through voltage or frequency excursions or other means [9], [10]) and disconnect. As a consequence of this, most islanding results in a loss of supply. While this is clearly appropriate in the current situation where DG is isolated and penetration is low, it is really not tenable in a possible future situation in which the overall power system relies on a high DG penetration. In this scenario reliability considerations start to become dominant, and there will be substantial motivation to examine strategies that are based on sustaining islanded operation to gain one of the major benefits from distributed generation. This contribution seeks to examine a new methodology for optimal load shedding in anticipation of this future scenario. However, it is recognized that there are also issues related to system ownership and the point of common coupling which go beyond the strictly technical aspects tackled here. The possibility of allowing the system to segregate into self sustained islands is very appealing indeed. This philosophy could also pave the way for selfhealing technologies that could isolate stressed, faulty equipment while maintaining supply to other portions and avoiding cascading effects [11]. To a degree, the implementation of such operational philosophies is a daily issue for developing countries whose power systems suffer routine shortages. These systems are operated in ways to reduce the probability of system wide blackouts [12].

Most utilities have installed multiple frequency level load shedding schemes [13]. In this investigation, the development of optimal load shedding with dynamic constraints is being further enhanced through the use of an optimal load shedding time to further the corrective control of power systems and facilitate islanded operation. This contribution presents a sig- 
nificant enhancement to the optimization technique developed in [14]. The new procedure yields the optimal load shedding time as well as the load shedding amount. It also uses a global approach to drive a disturbed system towards a known stable operating point. New control variables and goal functions have also been implemented in this formulation. All algorithms assume centralized load shedding as well as a communication infrastructure to be available [15], [16]. Also, all observers and switching operations are assumed to be ideal.

\section{PREvious WORK}

The use of load shedding as a mitigation tool has been constantly evolving and different approaches have been formulated. Relaying schemes like under-frequency (UF) and rate of change of frequency (ROCOF) are some examples of the mechanisms implemented to trigger a load shedding event. Typical UF schemes trigger load shedding based on pre-configured frequency levels. Two or more shedding stages may exist depending on the utility philosophy. When the system frequency reaches a specified threshold value, a time delay is inserted prior to the shedding action to try to avoid over shedding and assist the coordination of the next stage. This technique is not without inherent complications. Too few frequency levels could lead to over-shedding, but, on the other hand, time delays between stages could add up and may not allow for enough load to be shed in time to reestablish nominal frequency. The implementation of ROCOF techniques mitigates some of these problems. It permits better estimation of the load-generation unbalance paving the way for schemes that could adapt the relay settings to a specific event. Cutoff frequencies as well as the amount of load to be shed have typically been determined based on an operator's experience and simplified power system models. Newer adaptive techniques have come to light to fulfill more complicated and challenging modern demands on power systems. These techniques are also constantly changing to accommodate newer technologies and operating philosophies [17], [18].

The optimal load shedding problem formulation has also been evolving. An early version stated the problem as a minimum load shedding problem [19]. In this approach, the goal was to find the minimum amount of load to shed while satisfying load flow equations and static constraints like line flows, voltage, angular limits and shedding constraints. The formulation has several advantages over conventional load flow. One of its strongest points is that it provides an optimization frame for distributing the slack between generation and load throughout the available nodes. This is particularly useful, especially after disturbances or equipment outages, when corrective action, other than rescheduling, might be necessary. Transmission limits could take the form of transfer limits or angle differences.

Load shedding constraints provide a suitable framework for restricting the maximum amount of load to be shed at a specific site. Critical loads like hospitals or prime customers can benefit from this capability. The weight imposed on each load shedding bus could also be adjusted to reflect a specific customer hierarchy, tariff, or any other customer differentiation method. Further improvements have come in the form of increased detail in the power system model. The approach reported in [20] takes into consideration machine governor droop and the frequency and voltage characteristics of loads.

The latest methods consider load flow restrictions as well as system dynamics before and after a load shedding operation has taken place. The desired effect is to capture the dynamic response of each load shedding operation and to detect the best load shedding option to minimize an objective function based on system quantities as well as other variables. Dynamic methods are based on the discretization of the system's state trajectories. After discretization, the dynamic state equations are treated as algebraic constraints in the optimization problem. These trajectories are squared and integrated to estimate the "cost" of an iteration and to obtain the next load shedding values that should further reduce the cost function. This is done until convergence is achieved.

Application of a load shedding action as fast as physically possible [21] and at arbitrary, predetermined time steps [22] have been reported. The optimal load shedding time, to the best of the authors' knowledge, has never been suggested or used before. The current trend is to apply all corrective measures as soon as possible or delayed only for the sake of event discrimination. However, simulation results show that increased damping and enhanced recovery trajectories are observed when the corrective action is applied at the optimal time.

\section{Partitioned Explicit(PE) Method}

In the Partitioned Explicit (PE) method the set of algebraic equations (2) are decoupled from the transition function $\mathrm{H}$, and all system algebraic equations are now treated as a separate group of constraints in the optimization problem.

Consider a power system represented in the compact form,

$$
\begin{aligned}
\dot{x} & =f(x, y) \\
0 & =g(x, y, u)
\end{aligned}
$$

Defining $H$ as the transition equation, in its continuous form it must satisfy,

$$
H=\dot{x}-f(x, y)=0
$$

Applying the trapezoidal rule to (3) yields the discretized transition equation, $H_{k}$,

$$
H_{k}=x^{k+1}-x^{k}-\frac{\Delta t}{2}\left[f\left(x^{k+1}, y^{k+1}\right)+f\left(x^{k}, y^{k}\right)\right]
$$

The cost function is given by:

$$
C(x, y, u)=C_{1}(x)+C_{2}(y)+C_{3}(u)
$$

where

$$
\begin{gathered}
C_{1}(x)=\left(x-x^{*}\right)^{T} Q \Delta t\left(x-x^{*}\right) \\
C_{2}(y)=\left(y-y^{*}\right)^{T} Q \Delta t\left(y-y^{*}\right) \\
C_{3}(u)=P_{L S}^{2}\left(t_{f}-t_{1}\right)
\end{gathered}
$$

The optimization problem is:

$$
\text { minimize } C(x, y, u)
$$


subject to

$$
\begin{aligned}
& H_{k}(x, y)=0 \\
& g(x, y, u)=0
\end{aligned}
$$

Using Lagrangian multipliers, the augmented cost function is given by:

$$
L=C_{1}(x)+C_{2}(y)+C_{3}(u)+\gamma^{T} H_{k}(x, y)+\beta^{T} g(x, y, u)
$$

First Order Necessary Conditions (FONC) :

$$
\begin{gathered}
\frac{d L}{d x}=\frac{d C_{1}}{d x}+\gamma^{T} \frac{d H}{d x}+\beta^{T} \frac{d g}{d x}=0 \\
\frac{d L}{d y}=\frac{d C_{2}}{d y}+\gamma^{T} \frac{d H}{d y}+\beta^{T} \frac{d g}{d y}=0 \\
\frac{d L}{d u}=\frac{d C_{3}}{d u}+\beta^{T} \frac{d g}{d u}=0 \\
\frac{d L}{d \gamma}=H_{k}(x, y)=0 \\
\frac{d L}{d \beta}=g(x, y, u)=0
\end{gathered}
$$

\section{A. PE Algorithm}

The mathematical problem at hand has several aspects. First, the overall problem is best described as a constrained, nonlinear mathematical programming problem. The goal is to minimize system voltage and frequency oscillations following a disturbance with an optimal corrective load shedding action. This problem requires an iterative solution. Each iteration of the optimization problem controls all subsequent stages of the algorithm. Second, the nonlinearities of the system trigger the use of discretization techniques to estimate the dynamics of the system. These discretized trajectories are later used, each time-step, as equality constraints for the overall optimization routine to estimate the cost of the current iteration. Third, the time domain simulations require the simultaneous solution of state equations and algebraic network interface equations. Transmission grid as well as load characteristics and system voltages are all part of the algebraic set of variables that describe the network.

All the elements previously described are found within the algorithms developed to solve the optimization problem. The well-known lambda search gradient method [23] has been implemented and acts as the main engine of the algorithm.

\section{B. Proposed solution}

The goal of the algorithm is to iteratively find the input that minimizes (5). The cost computation requires the time domain simulation of the islanded section of the grid from the time immediately after clearing the fault until a final time. This final time must be long enough to capture all the dynamics but also, as short as possible to reduce the order of the system.

Fig. 1 shows a conceptual flowchart of the PE method. Before iterating to find the optimal input, several actions must take place. A stable initial condition $\left[x_{o} y_{o}\right]$ is extracted from a steady state solution to (1) and (2). The system is

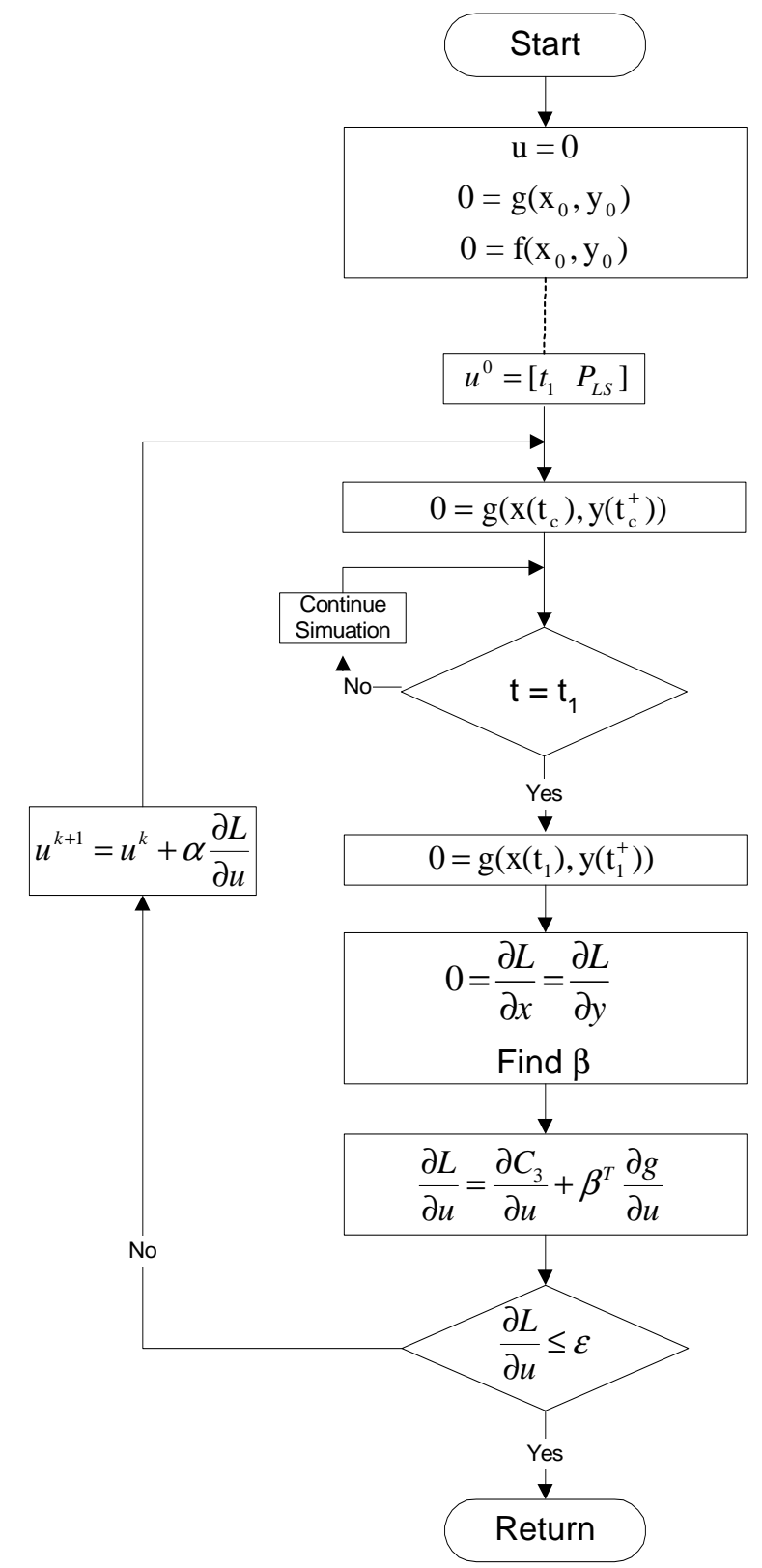

Fig. 1. PE method conceptual diagram.

then disturbed and the condition of the system following the formation of the island is saved and subsequently used as the initial condition for the time domain simulations of the response to every input. The input $u^{k}$ contains two quantities, the load shedding time $t_{1}$ and the vector $P_{L S}$. The simulation progresses until $t=t_{1}$ at which time the load shedding occurs. Once the load has been shed, the simulation continues to its end.

Once the simulation has concluded, the lambda search gradient method calls for the calculation of the Lagrangian multipliers, particularly $\beta$. The linear system given by (6) and (7) must be solved. Given the size of the problem it is very advisable to exploit sparsity. Some of the partial derivatives involved are shown below.

Partial derivatives with respect to $x$ are given by: 


$$
\frac{d H}{d x}=\left|\begin{array}{ccccc}
\frac{d H_{1}}{d x_{1}} & \frac{d H_{1}}{d x_{2}} & 0 & \ldots & 0 \\
0 & \frac{d H_{2}}{d x_{2}} & \frac{d H_{2}}{d x_{3}} & \ldots & 0 \\
\vdots & \vdots & \vdots & & \vdots \\
0 & 0 & 0 & \ldots & \frac{d H_{n_{T}}}{d x_{n_{T}}}
\end{array}\right|
$$

where,

$$
\begin{gathered}
\frac{d H_{j}}{d x_{j}}=-I-\frac{\Delta t}{2} \frac{d f\left(x_{k}, y_{k}\right)}{d x_{k}} \\
\frac{d H_{j}}{d x_{j+1}}=I-\frac{\Delta t}{2} \frac{d f\left(x_{k+1}, y_{k+1}\right)}{d x_{k+1}}
\end{gathered}
$$

with respect to $y$ :

$$
\begin{gathered}
\frac{d H}{d y}=\left|\begin{array}{ccccc}
\frac{d H_{1}}{d y_{1}} & \frac{d H_{1}}{d y_{2}} & 0 & \ldots & 0 \\
0 & \frac{d H_{2}}{d y_{2}} & \frac{d H_{2}}{d y_{3}} & \ldots & 0 \\
\vdots & \vdots & \vdots & & \vdots \\
0 & 0 & 0 & \ldots & \frac{d H_{n_{T}}}{d y_{n_{T}}}
\end{array}\right| \\
\frac{d H_{j}}{d y_{j}}=-\frac{\Delta t}{2} \frac{d f\left(x_{k}, y_{k}\right)}{d y_{k}} \\
\frac{d H_{j}}{d y_{j+1}}=-\frac{\Delta t}{2} \frac{d f\left(x_{k+1}, y_{k+1}\right)}{d y_{k+1}}
\end{gathered}
$$

With the current formulation it is not necessary to differentiate the algebraic equations with respect to future or past terms $k-1$ or $k+1$. These equations are no longer part of the transition equation (4). The algebraic constraints are incorporated as follows.

$$
\frac{d g}{d x}=\left|\begin{array}{cccc}
\frac{d g_{1}}{d x_{1}} & 0 & \ldots & 0 \\
0 & \frac{d g_{2}}{d x_{2}} & \ldots & 0 \\
\vdots & \vdots & & \vdots \\
0 & 0 & \ldots & \frac{d g_{n_{T}}}{d x_{n_{T}}}
\end{array}\right|
$$

Similarly with respect to $y$ :

$$
\frac{d g}{d y}=\left|\begin{array}{cccc}
\frac{d g_{1}}{d y_{1}} & 0 & \ldots & 0 \\
0 & \frac{d g_{2}}{d y_{2}} & \ldots & 0 \\
\vdots & \vdots & & \vdots \\
0 & 0 & \ldots & \frac{d g_{n_{T}}}{d y_{n_{T}}}
\end{array}\right|
$$

After evaluating (12) and (13) the resulting matrices are not necessarily square, the number of dynamic states, $n_{x}$, may differ from $n_{y}$, the number of algebraic variables. Fortunately (11) and (14) yield square matrices.

Rearranging (6),

$$
\gamma^{T}=-\frac{\partial C_{1}}{\partial x} \frac{\partial H^{-1}}{\partial x}-\beta^{T} \frac{\partial g}{\partial x} \frac{\partial H}{\partial x}^{-1}
$$

Substituting (6) into (7) and solving for $\beta^{T}$ yields

$\beta^{T}=\left[\frac{\partial C_{2}}{\partial y}-\frac{\partial C_{1}}{\partial y} \frac{\partial H}{\partial x}^{-1} \frac{\partial H}{\partial y}\right] \cdot\left[\frac{\partial g}{\partial x} \frac{\partial H^{-1}}{\partial x} \frac{\partial H}{\partial y}-\frac{\partial g}{\partial y}\right]^{-1}$

An alternate formulation may be expressed as:

$$
-\left[\begin{array}{c}
\frac{\partial C_{1}}{\partial x} \\
\frac{\partial C_{2}}{\partial y}
\end{array}\right]=\left[\begin{array}{cc}
\frac{\partial H}{\partial x} & \frac{\partial g}{\partial x}^{-1} \\
\frac{\partial H}{\partial y} & \frac{\partial g}{\partial y}^{-1}
\end{array}\right]^{T}\left[\begin{array}{l}
\gamma \\
\beta
\end{array}\right]
$$

Once $\beta$ has been updated, (8) is evaluated and tested for a stationary point. If its norm approaches zero, the current input is the optimal solution, otherwise the input is updated according to $u^{k+1}=u^{k}+\alpha^{k} \frac{d L}{d u}$. A new iteration would once again begin by initializing the system to the fault cleared state and the process repeated until convergence.

\section{Test CASES}

The algorithms have been explored by application to test cases. The objective here is to test all the algorithms and to validate, and document results.

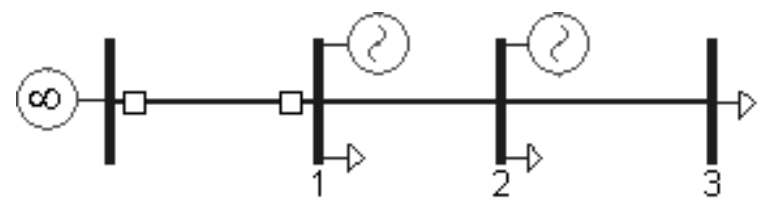

Fig. 2. Simple DG system shown with its interconnection to the utility power system.

A single-line diagram for a test power system is shown in Fig. 2. The scenario simulated with this system is representative of a simple, grid-connected, DG system. Before the disturbance, the utility and the DG system are tied together. The DG system is assumed to be importing some power from the utility. A three-phase fault occurs in the tie line and after the fault is cleared a DG island is formed. Prior to the disturbance, the island was importing power and, after it, excess load exists.

During the disturbance the system voltage drops, load decreases and generators start accelerating. Once the fault is cleared, an island is formed, its voltage starts to recover and excess load exists. The main idea behind the optimal load shedding time is to make use of this excess load to quickly slow down the generators, and optimally shed the excess load once the island approaches synchronous speed.

Several one-dimensional and multi-dimensional examples have been completed. Simplified conditions facilitate the use of two and three-dimensional plots to illustrate the various stages of the problem. Their outlining assumptions and preliminary results are presented below. 


\section{A. Reduced dimension visualization}

One-dimensional examples, despite their lack of practical application, are useful to enhance visualization of the problem. They provide the best insight into the challenges to be tackled as well as visual evidence of the behavior of the algorithms. Several one and two dimension examples were tested. The goal was to test the performance of the optimization algorithm individually for each component.

1) Optimal load shedding: In the first example, the goal was to test the load shedding portion of the optimization algorithm. To achieve this, several simplifications were implemented. Only bus \#1 is assumed capable of shedding load and all load will be shed at a pre-determined load shedding time. Combined, these two conditions simplify the problem into a one-dimensional optimal load shedding problem.

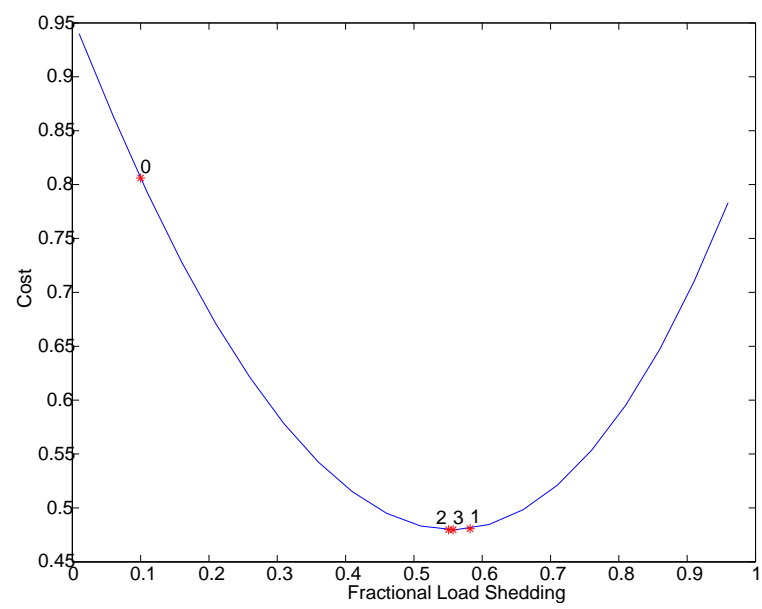

Fig. 3. One-dimensional iterations with PE method.

In Fig. 3, a sample plot of the cost vs. the fraction of load shed is shown. The numbers along the curve depict the iterations followed by the optimization algorithm using the PE method. It can be seen that only three iterations were needed on this particular test.

The second example is similar to the first one, except that this time busses \#1 and \#3 are both capable of shedding load simultaneously at an arbitrary time.

Fig. 4 shows a set of contour lines of (5) vs. the fractional amount of load shed at busses \#1 and \#3. On this occasion 14 iterations were necessary to achieve convergence.

2) Optimal shedding time: At this point, the optimal time portion of the optimization algorithm is also evaluated. On this occasion the algorithm tries to determine the best time to shed a fixed amount of load at bus \#1. The results of this test are presented in Fig. 5.

\section{B. Time optimal load shedding}

The full capability of the algorithm is now demonstrated. The output contains the optimal time to shed load as well as the optimal amount.

Fig. 6 shows the contour lines of (5) plotted on a shedding time vs. the load shedding fraction, y, axis. The algorithm converged after 5 iterations.

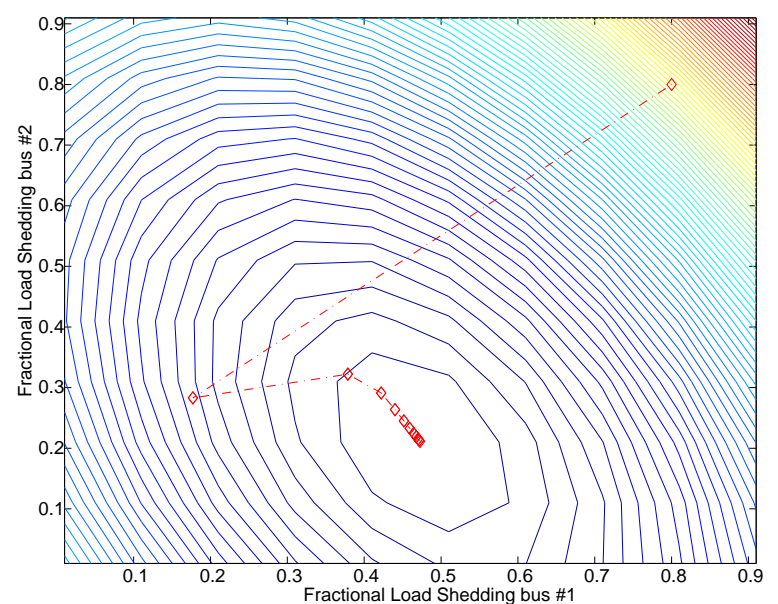

Fig. 4. Two busses, optimal load shedding at a fixed time.

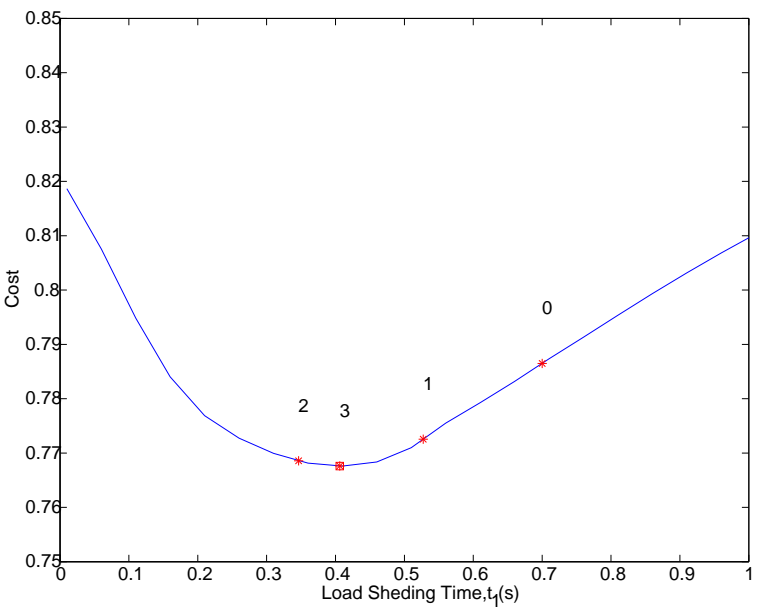

Fig. 5. Shedding of a fixed amount of load at the optimal time.

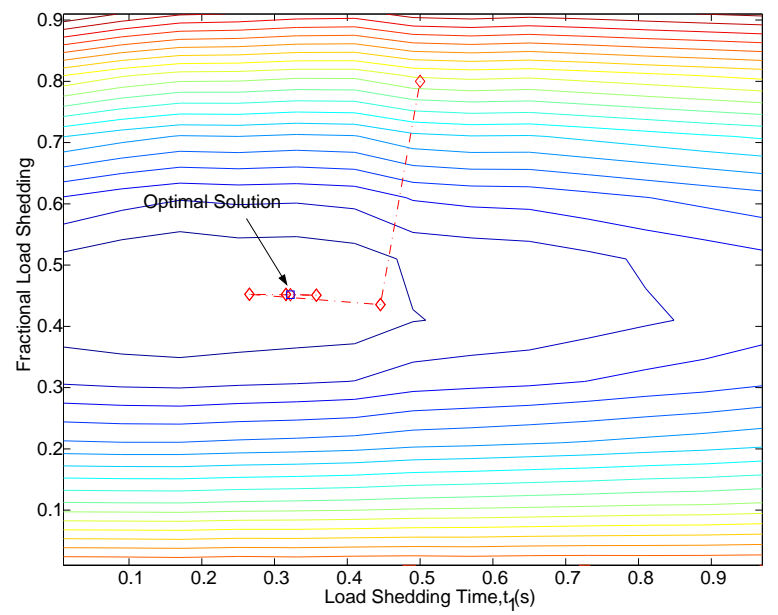

Fig. 6. Single bus optimized load shedding time and amount.

In the final test, shown in Fig. 7, the system has the capability to shed load at busses \#1 and \#2. The performance of the algorithm is shown as a function of the iteration number. Despite the twenty iterations to meet the stopping criteria, it can be seen that the cost and all other variables stabilize after only ten iterations. On this test, the total cpu time on a $2.8 \mathrm{GHz}$ 

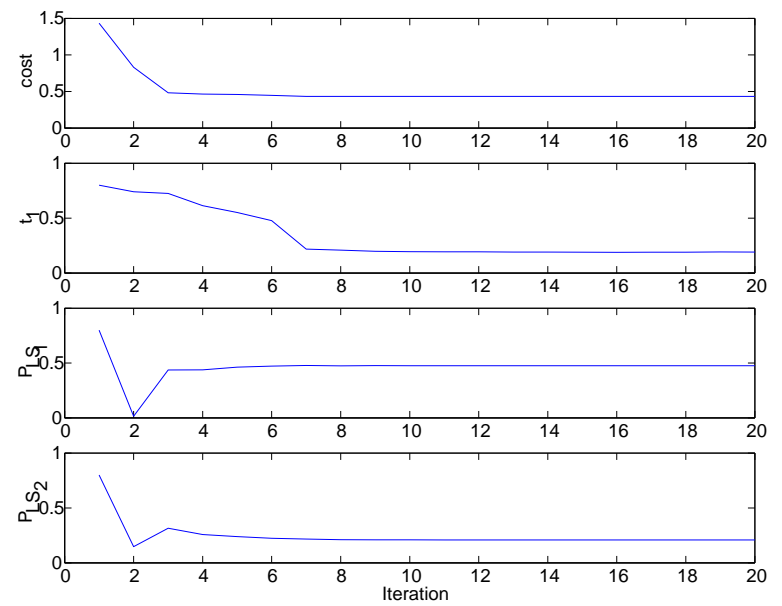

Fig. 7. Two busses optimized load shedding time and amount.

machine was 82 seconds.
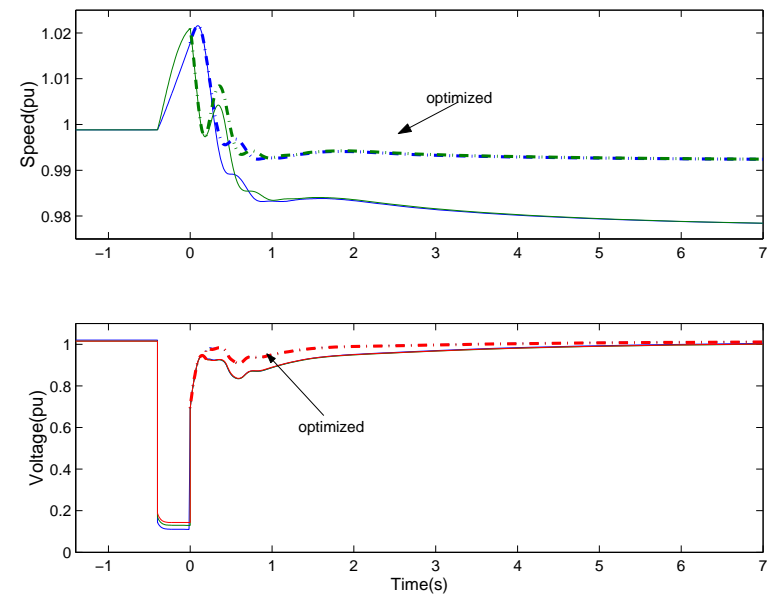

Fig. 8. Speed and voltage response.

Fig. 8 shows the dynamic response to the application of the time optimal load shedding under the conditions previously described in the final test case. It provides what might be the best insight into the operation of the algorithm.

In the algorithm used to evaluate all previous cases, the weights applied to speed and voltage oscillations were eleven times higher than all others. Under those conditions, the goal becomes to find an input that minimizes primarily speed and voltage deviations. According to Fig. 7, the optimal time to shed load was about 0.19 seconds after clearing the fault. Closer inspection of Fig. 8 reveals the inner workings behind the solution. With respect to the voltage, this point occurs almost immediately after the voltage peaked following the swing produced by the removal of the fault. Therefore, the braking torque produced by the load peaked and has already produced the maximum breaking torque. Given the "droopy" characteristic of the island, after this point the excess load becomes a "command" to settle at a new, lower, speed to compensate for the excess load. Shedding at this time uses the excess load to brake the machines following the fault and almost completely avoids under-frequency operation.

Meanwhile, the dominant machine has already lost enough momentum to decelerate beyond synchronous speed and the smaller, "follower", machine at bus \#2 has reached synchronous speed and it is just about ready to start accelerating again. The algorithm detects that shedding prior to this point will reduce the braking torque and prolong the oscillation. Furthermore, shedding after this point would not significantly alter the trajectory of the dominant machine, sending it to an under-frequency condition likely to prolong the oscillations. Therefore, it was found that 0.19 seconds was the optimal time in this instance.

\section{Discussion \& CONCLUSIONS}

The methodology developed represents a significant enhancement to the disciplines of corrective control and islanding for distributed power systems. Compared to early optimal load shedding approaches, the algorithm incorporates the most significant missing feature, dynamic trajectories of the system. With respect to newer methods, the optimal load shedding time translates into more efficient and effective use of a shedding scheme.

The optimization formulation is both flexible and robust. It can handle a variety of conditions implemented either through the goal function or the power system model. With modifications, the algorithms could also handle a variety of scenarios like multiple islands or an optimal load shedding schedule, where more than one shedding stage could be determined.

The incorporation of semiconductor inverters (required for asynchronous generators and most renewable energy sources) creates a very different set of circumstances and, is a priority. Given the close ties between the dominant machine and the optimal time, it is likely that heavy penetration of very fast devices will reduce the optimal shedding time to a point at which it becomes ineffective.

\section{Vi. Practical Implementation}

Although this treatment has been designed to outline the principle of the proposed algorithm, several other practical aspects must be considered prior to any implementation. First, it must be recognized that all optimization methods which anticipate large matrices are computationally intensive. It is not envisaged that this scheme would be run on-line, but rather run in the background to generate parameter performance envelopes (look-up tables) which would then be used for fast implementation. Continuous background envelope refreshment is needed to account for load and configuration changes.

As was indicated in Section 1, it is anticipated that an overlaid communication network exists. However, although not yet fully evaluated, the indications are that the algorithm is tolerant to corrupt or missing data, particularly if it is not associated with a dominant machine. Further work is ongoing to examine the parameter sensitivities and to evaluate the communication bandwidth needed for proper implementation.

\section{ACKNOWLEDGEMENTS}

The Authors are indebted to the University of Puerto Rico for the support of this work. Gratitude is also expressed to the Grainger Foundation and to the Gates Millennium Scholarship Foundation for the provision of awards. 


\section{REFERENCES}

[1] R. Brown and L. Freeman, "Analyzing the reliability impact of distributed generation," in Proc. IEEE Power Engineering Society Summer Meeting (PESSM'2001), vol. 2, Vancouver, BC Canada, July 2001.

[2] F. Edwards, G. Dudgeon, J. McDonald, and W.E.Leithead, "Dynamics of distribution networks with distributed generation," in Proc. IEEE Power Engineering Society Summer Meeting (PESSM'2000), vol. 2, Seattle, WA USA, July 2000.

[3] P. O'Kane, B. Fox, and D. Morrow, "Impact of embedded generation on emergency reserve," IEE Proceedings on Generation, Transmission and Distribution, vol. 146, no. 2, Mar. 1999.

[4] J. Slootweg and W. Kling, "Impacts of distributed generation on power system transient stability," in Proc. IEEE Power Engineering Society Summer Meeting (PESSM'2002), vol. 2, Chicago, WI USA, July 2002.

[5] N. Ijumba, A. Jimoh, and M. Nkabinde, "Influence of distribution generation on distribution network performance," in Proc. IEEE (AFRICON'1999), vol. 2, Cape Town, South Africa, Sept. 1999.

[6] L. Kojovic and R. Willoughby, "Integration of distributed generation in a typical usa distribution system," in Proc. CIRED 16th International Conference and Exhibition on Electricity Distribution (IEE Conf. Publ), vol. 4, no. 482, here, June 2001.

[7] R. Walling and N. Miller, "Distributed generation islanding-implications on power system dynamic performance," vol. 1, Chicago, WI USA, July 2002.

[8] Standard for Interconnecting Distributed Resources with Electric Power Systems, IEEE Std. 1547, 2003.

[9] D. Rajicic, "A method for determination whether a network is connected or split," IEEE Power Eng. Rev., vol. 21, no. 7, July 2001.

[10] J. Motohasi, T. Ishikawa, and C. Nakazawa, "Comparison of digital simulation and field test results of islanding detection systems for synchronous generators," in Proc. IEEE Power Engineering Societe Winter Meeting (PESWM'1998), vol. 2, Tampa, FL USA, Feb. 1998.

[11] M. Amin, "Toward self-healing energy infrastructure systems," IEEE Comput. Appl. Power, vol. 14, no. 1, Jan. 2001.

[12] S. Ahmed, N. Sarker, A. Khairuddin, M. Ghani, and H. Ahmad, "A scheme for controlled islanding to prevent subsequent blackout," IEEE Trans. Power Syst., vol. 18, no. 1, Feb. 2003.

[13] A. Ait-Kheddache and S. Ebron, "Optimal load shedding methodologies in power systems," in Proc. IEEE Southeastcon, Knoxville, TN USA, Apr. 1988

[14] M. L. Scala, M. Trovato, and C. Antonelli, "On-line dynamic preventive control: an algorithm for transient security dispatch," IEEE Trans. Power Syst., vol. 13, no. 2, 1998.

[15] G. Heydt, C. Liu, A. Phadke, and V. Vittal, "Solution for the crisis in electric power supply," 2001.

[16] T. Taylor, H. Willis, and M. Engel, "New considerations for distribution network planning and design," in Proc. IEE 14th International Conference and Exhibition on Electricity Distribution, vol. 6, no. 438, Birmingham, UK, 1997.

[17] C. Bright, "Corocof: comparison of rate of change of frequency protection. a solution to the detection of loss of mains," in Proc. IEE Seventh International Conference on Developments in Power System Protection, Amsterdam, Netherlands.

[18] P.M.Anderson and M. Mirheydar, "An adaptive method for setting underfrequency load shedding relays," IEEE Trans. Power Syst., vol. 7, no. 2, May 1992.

[19] L. P. Hajdu, J. Peschon, T. Tinney, and D. Piercy, "Optimal load shedding policy for power systems," IEEE Trans. Power App. Syst., vol. 8, no. 3, Mar. 1968.
[20] K. Palaniswamy, J. Sharma, and K. Misra, "Optimum load-shedding taking into account of voltage and frequency characteristics of loads," IEEE Trans. Power App. Syst., vol. 104, no. 6, June 1985.

[21] E. D. Tuglie, M. Dicorato, M. L. Scala, and P. Scarpellini, "A corrective control for angle and voltage stability enhancement on the transient timescale," IEEE Trans. Power Syst., vol. 15, no. 4, Nov. 2000.

[22] D. Xu and A. Girgis, "Optimal load shedding strategy in power systems with distributed generation," in Proc. IEEE Power Engineering Society Winter Meeting (PESWM'2001), Columbus, OH USA.

[23] Power generation operation and control, 2nd ed. Wiley Interscience, 1996.

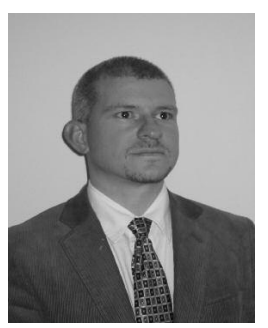

Erick E. Aponte (Student'98) was born in Hato Rey, Puerto Rico. He received the degree of B.Sc(Eng) from the University of Puerto Rico Mayaguez Campus in 1997 and his M.E. from the Rensselaer Polytechnic Institute in 1998. Erick holds an instructor position at UPRM since 1998, where he has been on leave of absence since August 2000 for his doctoral studies at RPI. His research interests are mainly power system dynamics, corrective control and distributed generation.

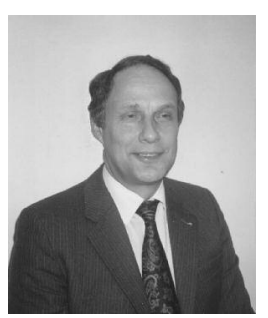

J. Keith Nelson (Fellow'90) was born in Oldham, UK and received his B.Sc(Eng) and Ph.D degrees from the University of London. He is currently Philip Sporn Chair of Electric Power Engineering at the Rensselaer Polytechnic Institute. Prior to his appointment at Rensselaer, he was manager of Electric Field Technology Programs at the General Electric R\&D Center in Schenectady, NY. He has held numerous IEEE appointments including that of the Presidency of the Dielectrics \& Electrical Insulation Society, 1995-6. He is a chartered electrical engineer and the recipient of the IEEE Millennium Medal. 